Paper

\title{
Effective thermal modeling of a thin film snubber resistor for power modules
}

\author{
Ryosuke Watanabe $^{1}$, Keita Izawa ${ }^{2}$, Shota Kajiya ${ }^{3}$, \\ Daiki Tsunemoto ${ }^{3}$, Koki Kasai ${ }^{1}$, Atsushi Kurokawa ${ }^{1}$, \\ and Toshiki Kanamoto ${ }^{1 \text { a) }}$ \\ ${ }^{1}$ Graduate School of Science and Technology, Hirosaki University \\ 3 Bunkyo-cho, Hirosaki-shi, Aomori 036-8561, Japan \\ ${ }^{2}$ Nikkohm co., Ltd. \\ 3-31 Minami-cho, Misawa-shi, Aomori 033-0036, Japan \\ ${ }^{3}$ Faculty of Science and Technology, Hirosaki University \\ 3 Bunkyo-cho, Hirosaki-shi, Aomori 036-8561, Japan \\ a)kana@hirosaki-u.ac.jp
}

Received July 31, 2019; Revised November 18, 2019; Published April 1, 2020

\begin{abstract}
This paper proposes an effective thermal compact modeling method for a thin film resistor that is dedicated for snubber circuits in power electronic modules. The objective compact model consists of thermal capacitors and thermal resistors. Some of the objective compact models express the effects of local air gaps as well as convective boundary conditions, which are natively non-linear phenomena. To identify their values, we use the surface temperature response of the thin film resistor to the input power. The experimental results show that the derived compact thermal model robustly causes the measured thermal durability to wide input power pulse width variations to reappear.
\end{abstract}

Key Words: thermal, thin, film, resistor, snubber, power

\section{Introduction}

Insulated-gate bipolar transistors (IGBTs) as well as silicon carbide ( $\mathrm{SiC}$ ) metal oxide silicon (MOS) transistors are used in state-of-the-art high-power electronic converter systems including automotive motor controls. They achieve switching loss reduction due to the high speed operation, but they need to be accompanied by snubber circuits that suppress unwanted ringing in the operating voltage and current $[1-3]$.

Typically snubber circuits consist of resistors and capacitors. To keep the efficiency of the snubber circuit, it is desired to minimize the equivalent series inductance, which is parasitic to the snubber RCs [4]. As a solution, monolithic integration of the snubber RCs has been proposed, targeting relatively low power applications [5]. Regarding high power applications, here we treat a thin film resistor, which reduces the parasitic inductance by shortening the body length.

Snubber circuits are being treated as part of the power modules [6]. Therefore, thermal management 
is one of the indispensable structure design processes of the snubber resistor. In this paper, we propose a simple yet effective thermal model along with the corresponding modeling method.

Our basic modeling approach is to express the heat transfer of the objective device structure as an equivalent thermal circuit [7-10]. The equivalent circuit is based on the Cauer network [11-14], which is essentially a linear expression. However, the equivalent circuit model partially approximates two kinds of nonlinear components. One is the boundary condition, especially the convective boundary condition treated in this study [15-17]. The other is the colony of air gaps formed under the thin film resistor body. We have experimentally identified an approximation of air gaps by pulse durability tests $[18-20]$.

The rest of the paper is organized as follows. Section 2 describes the objective thin film resistor structure and then explains how the pulse durability tests give the thermal approximation of the air gap effects. Section 3 depicts our equivalent circuit model of the thin film resistor as well as the method to determine the thermal RC parameters. Section 4 shows a reproduction of the measured thermal durability for pulse width variations of the input power.

\section{Pulse durability of thin film resistor}

\subsection{Evaluated structure}

In this study, industrial thin film NiCr resistors are considered. NiCr films $150 \mathrm{~nm}$ in thickness are sputter deposited on alumina substrates. Copper sheet is on the rear side of the substrates, and are soldered onto the copper flanges. The components are molded by epoxy resin, and the resin is not in contact with the NiCr thin film. The exterior of the objective resistor is shown in Fig. 1.

Here, we assumed that the resistors are thermally destructed by the pulsed power injection. Figure 2 shows the obtained scanning electron microprobe (SEM, JXA-8230, JEOL Ltd.) image of the NiCr surface. Figure 2(a) shows the surface of the $\mathrm{NiCr}$ sheet of the resistor. Figure 2(b) shows the laser trimmed area of the resistor that is trimmed by thermal evaporation by high power laser illumination, and the figure indicates that the $\mathrm{NiCr}$ film is melted and the $\mathrm{NiCr}$ becomes spherical near the edge of the trimmed area. In Fig. 2(c), the injected power induced damaged area of the NiCr sheet is shown. The figure indicates that the spherical $\mathrm{NiCr}$ also exists in the destructed area of the resistor, and this leads to the prediction that the resistors are thermally damaged by the high pulsed power injection.

In addition, the damaged area of the resistors is mainly located at the center of the $\mathrm{NiCr}$ sheet. In the central area, the heat is expected to be stored, and the resistor tends to reach a high temperature. On the other hand, the edge of the NiCr films is close to the lead frame, which can release the heat promptly. Figure 3 shows that the resistor is destructed at the center of the $\mathrm{NiCr}$ film. These behaviors are supporting evidence that the resistors considered in this study are thermally destructed.

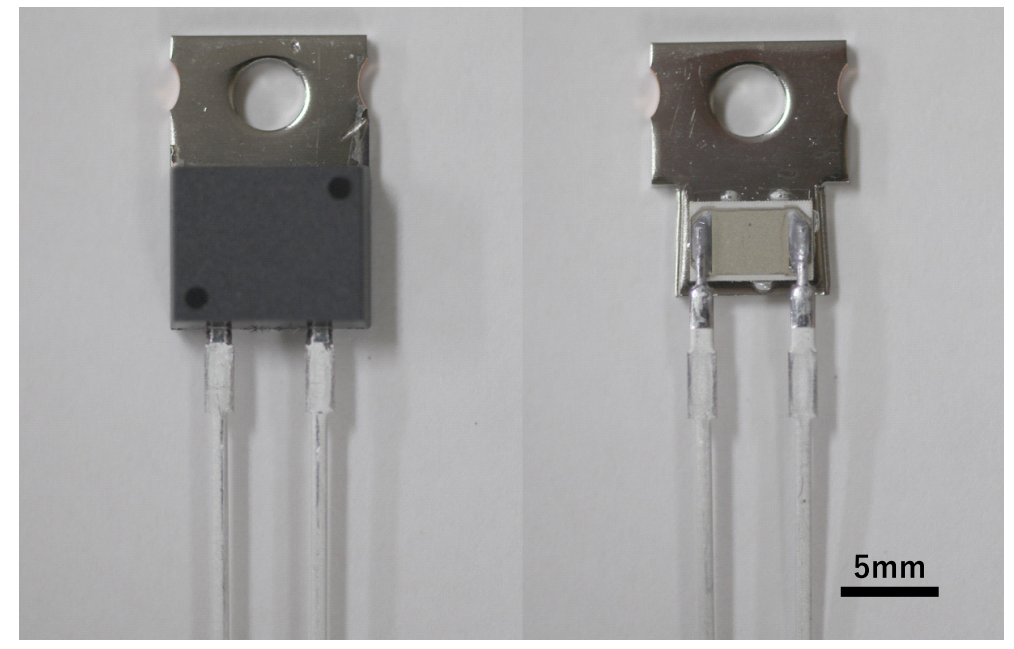

Fig. 1. Exterior of the thin film NiCr resistor. Left: The resistor is covered by epoxy resin. Right: The resistor without epoxy resin, and NiCr thin film can be seen in the center of the resistor. 
Thus, in the following paragraph, we would like to evaluate the thermal behavior of the NiCr thin film resistors to consider the destruction mechanism of the resistors.

To evaluate the thermal analysis of the thin film resistors, we prepare a technology computer-aided design (TCAD) model. To calculate the thermal behavior of the resistors, we use the finite element method (FEM) calculation software FEMTET Version 2018.1 (Murata Software Co., Ltd.) [22]. The thermal, construction, and electronic parameters of the resistor are set in the TCAD model, and an electro-thermal coupled transient analysis is performed. The prepared TCAD model is shown in
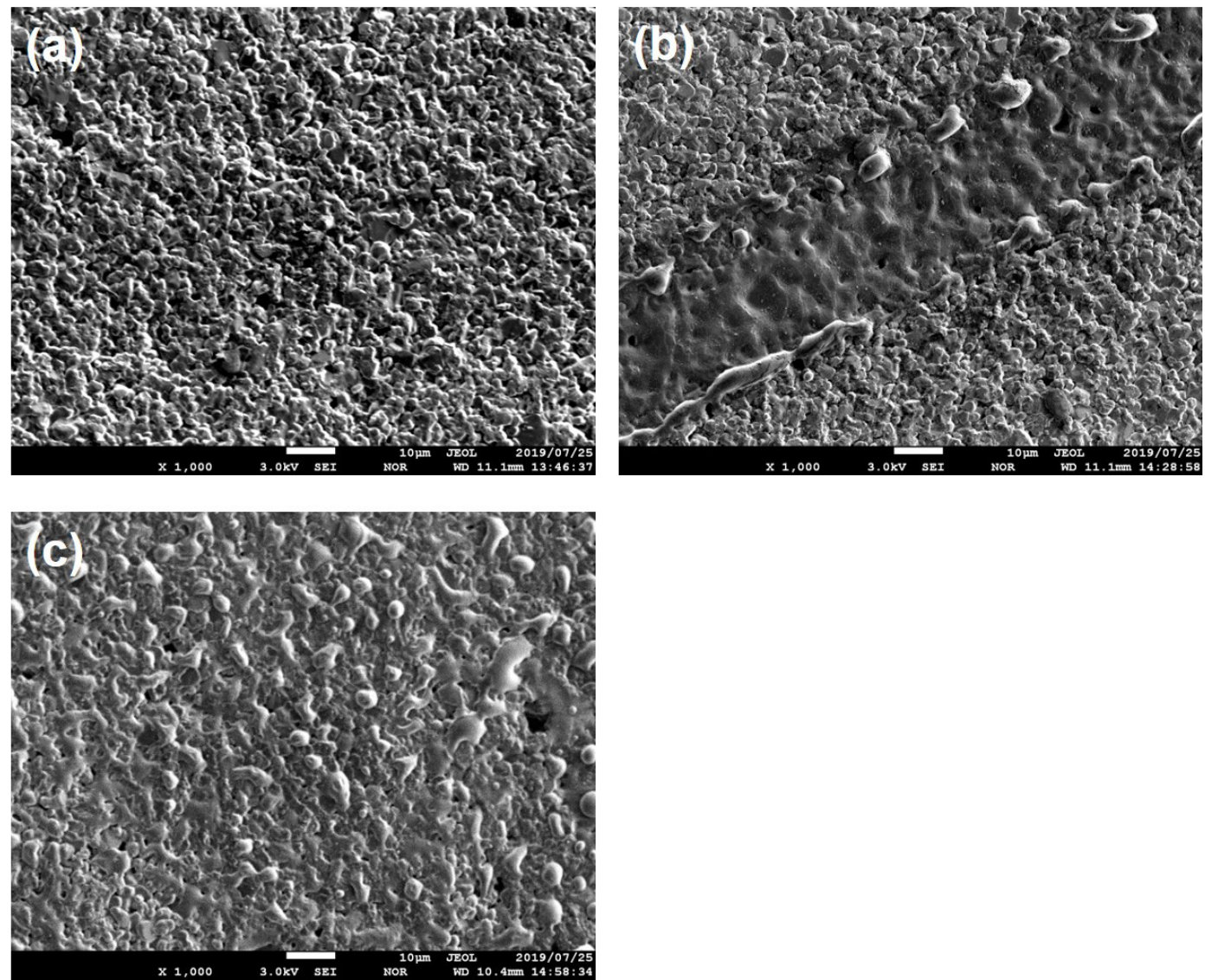

Fig. 2. SEM images of the $\mathrm{NiCr}$ thin film on the alumina substrate. (a) Surface of the NiCr thin film. (b) Laser trimmed area of the NiCr thin film. $\mathrm{NiCr}$ becomes spherical at the edge of the trimmed area. (c) Damaged area of the NiCr resistor. Spherical material exists here also.

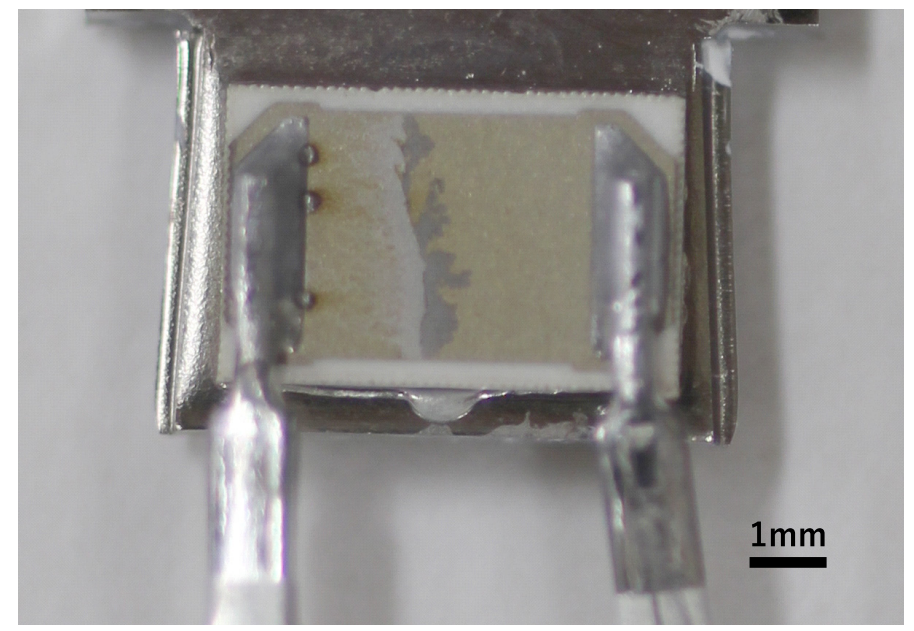

Fig. 3. Enlarged picture of the destructed $\mathrm{NiCr}$ thin film. Damaged area is located at the center of the $\mathrm{NiCr}$ film. 
Fig. 4. Dimensions of the considered TCAD model are also shown in the figure. An effective air gap $350 \mathrm{~nm}$ in width is introduced in the model (details are described later).

To fit the thermal boundary parameters of the TCAD model calculation, we compare the thermal time constant of continuous power responses for both the experiment and TCAD model calculation. First, we experimentally evaluate the thermal transient response of the resistors to obtain the thermal time constant and reached temperature of the experimental samples. The thermal transient response is evaluated by applying a continuous electric power to the resistor and measuring the thermal transient response with a thermographic camera (Fluke corp. Ti200). A schematic of the measurement system is shown in Fig. 5(a). In this constant power experiment, we need to measure the surface temperature of the NiCr films; thus, a bare $\mathrm{NiCr}$ resistor without epoxy resin is used. The temperature is measured at the center of the NiCr films. Figure 6(a) shows one of the experimentally obtained results and we record the temperature at the center of the $\mathrm{NiCr}$ thin films (white round mark).

Also, we performed the TCAD model calculation without epoxy resin to fit the thermal boundary parameters of the TCAD model calculation. Figure 6(b) indicates the temperature distribution of one of the TCAD model calculation results. We record the temperature at the center of the $\mathrm{NiCr}$ films (white round mark). In the experiment, the DUTs are in the ambient air as shown in Fig. 5(b). Transparent walls are placed merely for explosion proof. The backside is fully open. The ambient air is naturally convected. In our calculation, natural convective boundary condition is used in the TCAD model to be consistent with the experimental condition.

Experimentally obtained thermal transient behaviors are shown in Fig. 7. In this experiment, a constant power of $1.0 \mathrm{~W}$ is applied to the sample for 360 seconds, then the power is switched off to monitor the cooling behavior. The measured thermal time constant of the resistor is around 40 seconds. Also, the TCAD model calculation results in Fig. 7 reproduce the long time constant thermal behaviors after only the thermal boundary condition of the TCAD model is appropriately fixed. The above results indicate that the TCAD model calculation with an appropriate thermal boundary condition can reproduce enough of the thermal behavior of the resistors, and in the following section we will use the TCAD model calculation for the detailed thermal evaluations of the resistors.

\subsection{Pulse durability evaluation}

Next, we consider the effect of thermal isolation of NiCr thin films. Here, we introduce an effective air gap between the $\mathrm{NiCr}$ thin films and the alumina substrate in the TCAD model calculation.

Alumina sintered body substrate is used in the resistor. The surface of the alumina substrate obtained by SEM is shown in Fig. 8. This figure indicates that the surface is strongly roughened. Also, Fig. 2(a) indicates that the sputter deposited $\mathrm{NiCr}$ film is roughened. This means that the
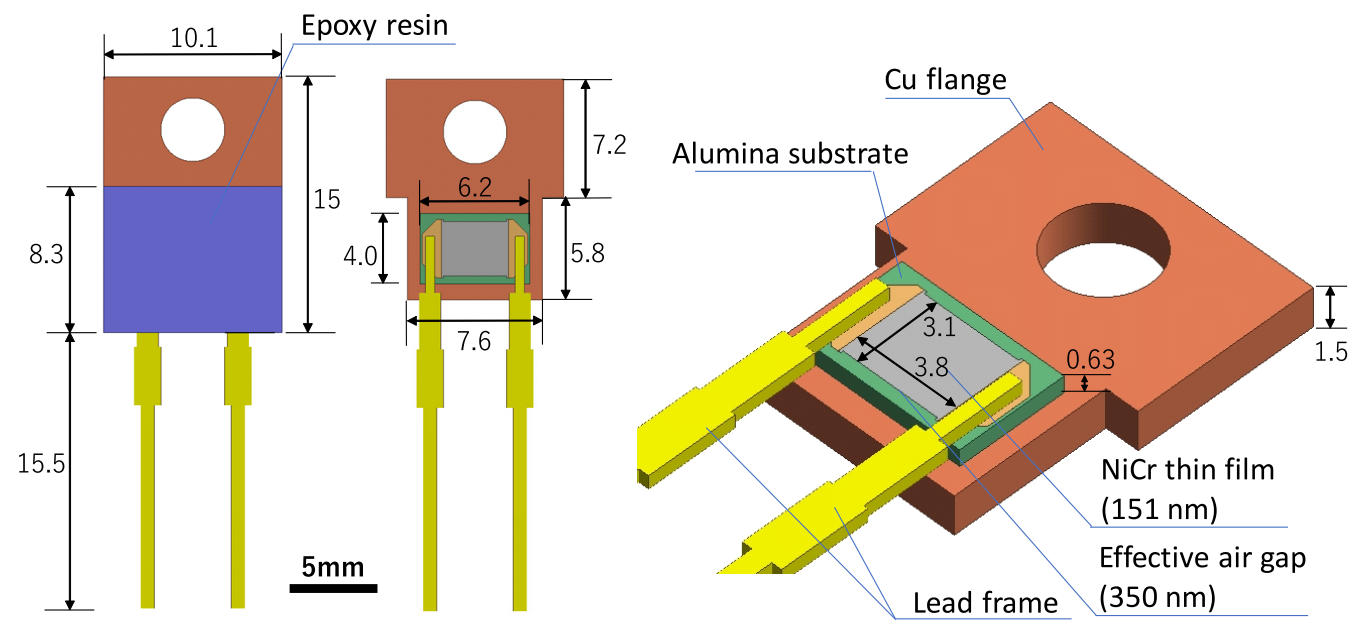

Fig. 4. Schematics of the considered NiCr thin film resistor. Dimensions are described in millimeter except NiCr film thickness and effective air gap width. Left: The resistor with epoxy resin. Center: The resistor without epoxy resin. Right: Enlarged figure of the resistor. 
$\mathrm{NiCr}$ sheet does not fully adhere to the alumina substrates or are deposited on the thermally isolated areas. The NiCr sheets are expected to be thermally isolated from the substrate. In this situation, the air gap inside the alumina substrate or between the alumina substrate and the $\mathrm{NiCr}$ thin film may cause the heat transfer from the $\mathrm{NiCr}$ films to the alumina substrates and the copper flange to be thermally resisted. The NiCr film is expected to reach a high temperature and finally is destructed. In the next step, we evaluate the thermal isolation effect of the $\mathrm{NiCr}$ thin film; however, it is difficult to reproduce the complete structure due to limited computational resources. Thus, in this study, we introduce the effective air gap in the calculation model. The effective air gap width dependence of the thermal behavior of the NiCr thin films is considered by numerically evaluating the melting behavior of the NiCr sheet.

To simplify the TCAD model calculation, we consider a simplified model where the NiCr thin film is on the alumina substrate with the effective air gap width. First, a constant power calculation is

(a)

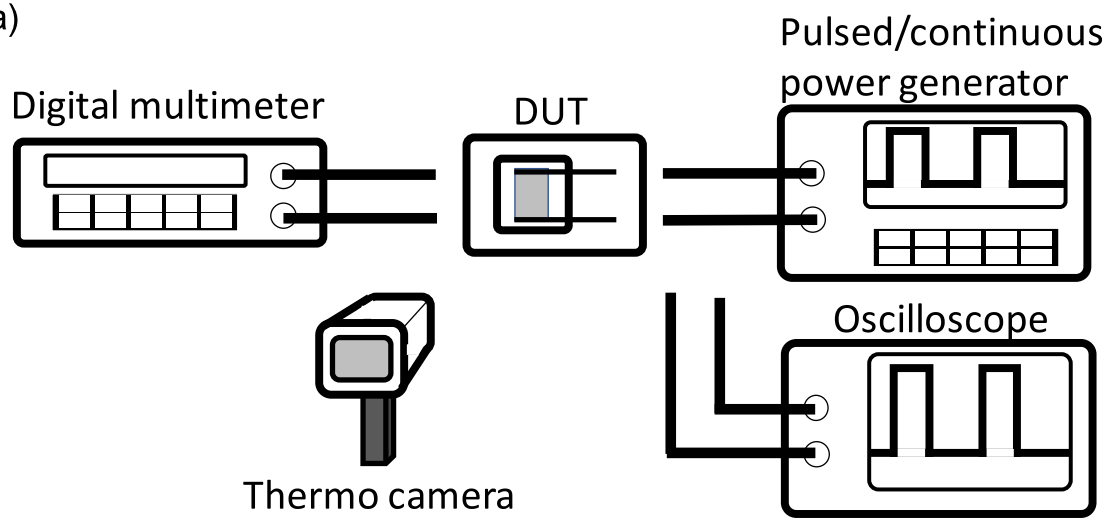

(b)

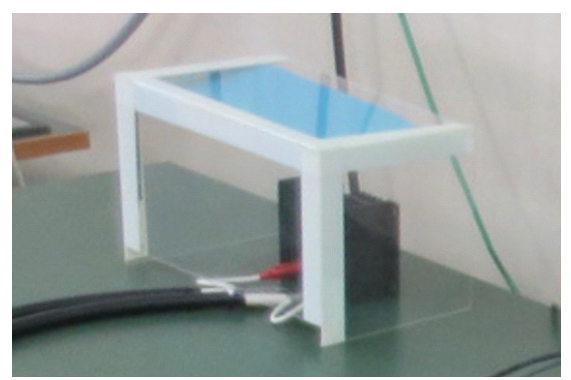

Fig. 5. Experimental measurement system. (a) Schematic of the system. Pulsed/constant power is applied to the resistor (DUT), and the surface temperature is measured by the thermographic camera. The resistivity of the resistors are checked with the digital multimeter. (b) Appearance of the DUT surroundings.

(a)

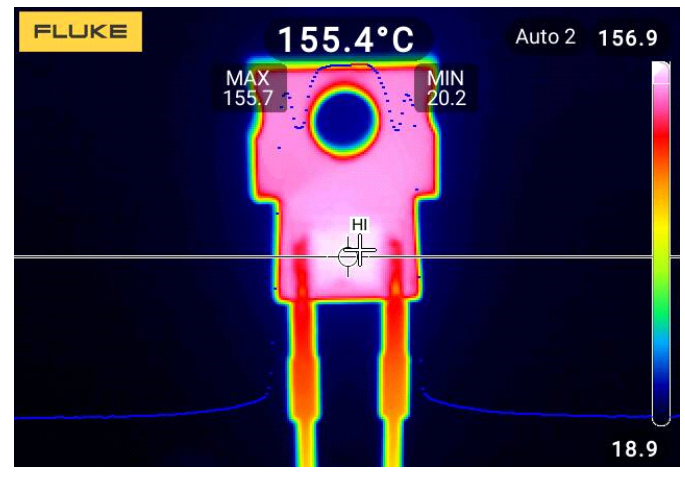

(b)

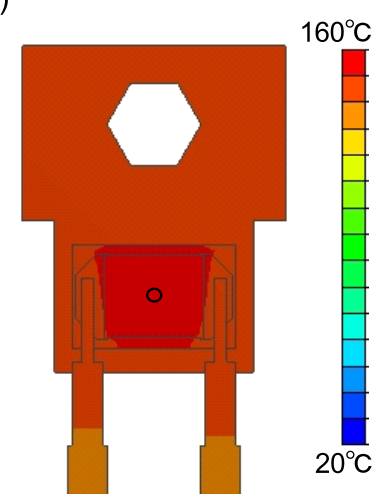

Fig. 6. Example of the temperature distribution of the DUT. Temperature is recorded at the center of the NiCr film (white round mark). (a) Experiment (b) TCAD model calculation 
carried out to see long time thermal behavior. Here, a constant power of $1.0 \mathrm{~W}$ is applied for 360 seconds, and then the power is switched off to measure the cooling behavior. The calculated results in Fig. 9 indicate that the effective air gap between the $\mathrm{NiCr}$ thin films and the alumina substrates hardly affects the long thermal time constant and the reached temperature of the resistor. When the $350 \mathrm{~nm}$ thick air gap is introduced, heat capacitance of the region is $3 \times 10^{-3} \mathrm{~J} / \mathrm{K}$ with thermal resistance of $1.25 \mathrm{~K} / \mathrm{W}$. Thermal time constant is $t=C R$, where $C$ and $R$ are heat capacitance and thermal resistance of the air gap, respectively. The thermal time constant of the air gap region is $3.75 \times 10^{-3}$ seconds. Because the thermal effect of the air gap is rapidly saturated, it does not affect the long-time thermal behavior. Thus, the existence of the air gap is difficult to find in the long-time thermal response experiment.

Next, the pulsed power injection condition of the resistor with the effective air gap is considered. The air gap width dependence of the reached temperature of the $\mathrm{NiCr}$ thin films evaluated by the TCAD model calculation is shown in Fig. 10. In this figure, the incident pulse width is fixed to

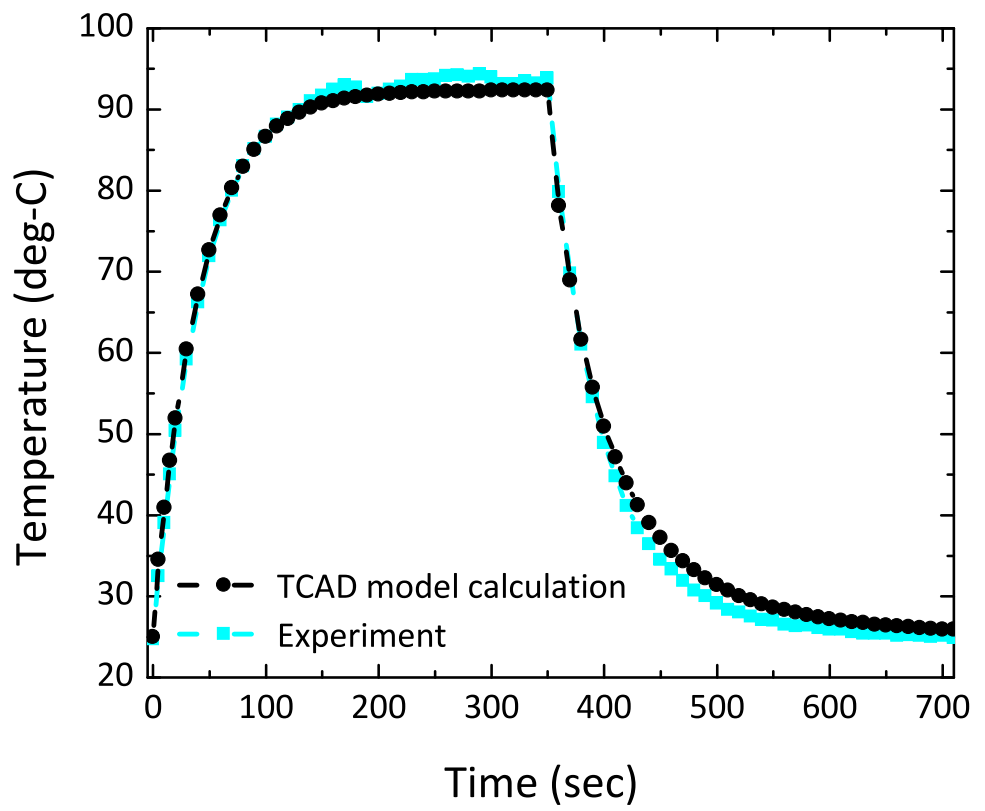

Fig. 7. Obtained thermal transient behavior of the resistor by experiment and TCAD model calculation.

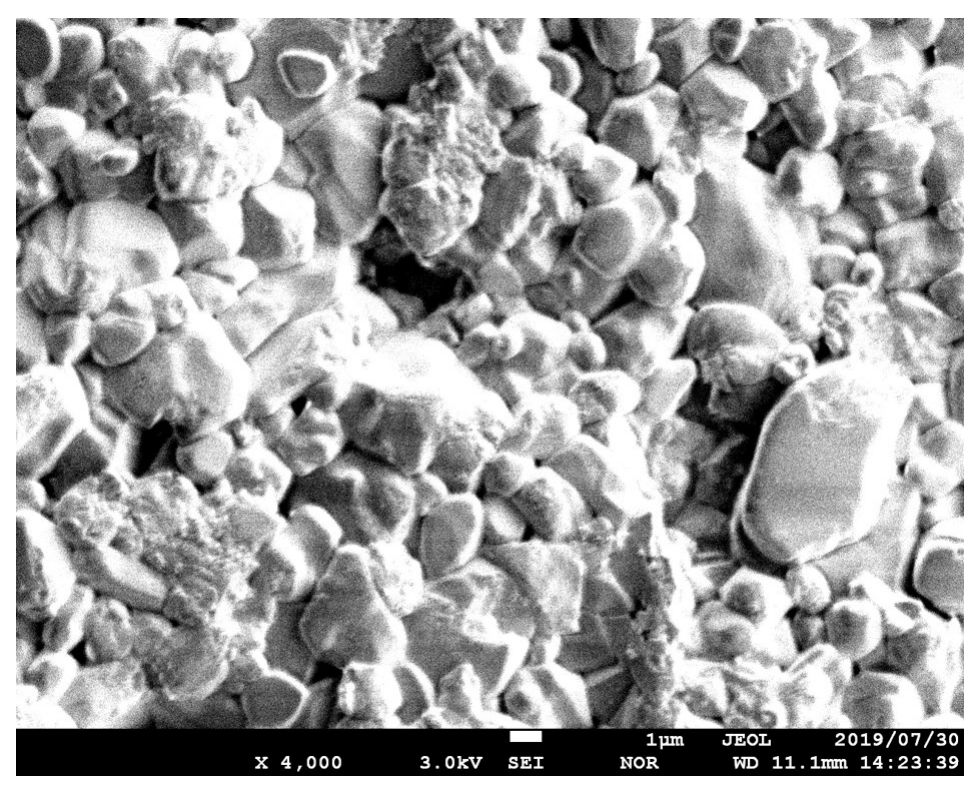

Fig. 8. SEM image of the surface of the alumina substrate. 
30 microseconds, the pulse period is $3 \mathrm{~ms}$, and the incident pulsed peak power is fixed to $1000 \mathrm{~W}$. Schematic of the pulse is shown in Fig. 11. The effective air gap width is modified from $0 \mathrm{~nm}, 100 \mathrm{~nm}$, $350 \mathrm{~nm}$, and 1 micrometer, respectively. Figure 10 clearly indicates that the width of the effective air gap strongly affects the reached temperature of the resistor for the pulsed power injection condition. In the periodically power injection condition, the peak power is quite high though pulse injection time (pulse width) is short. In this condition, averaged injection power is low (about 10W), but the air gap thermally follows the injected strong pulsed peak power because the air gap has small thermal time constant. When the sample has no air gap, the temperature of the $\mathrm{NiCr}$ films is slightly increased. On the other hand, for instance, when the width of the air gap between the $\mathrm{NiCr}$ thin film and the alumina substrate is 1 micrometer, the film temperature is drastically increased to around 2300 degrees, which is over the melting point of $\mathrm{NiCr}$ films (around 1400 degrees).

For the pulsed power injection situation, the TCAD model calculation revealed that the effective air gap between the $\mathrm{NiCr}$ thin films and the alumina substrates strongly affects the reached temperature of the $\mathrm{NiCr}$ sheet. The heat insulation behavior of the air gap causes heat to be stored inside the $\mathrm{NiCr}$ films. In this condition, although the average input power is low, the peak power of the input pulse can damage the $\mathrm{NiCr}$ sheet in a short time. We considered that the stored heat inside the $\mathrm{NiCr}$ sheet causes the very high temperature of the sheet within a few tens of microseconds and finally the sheet is melted and destructed.

\section{Thermal modeling of thin film resistor}

This section describes the proposed thermal equivalent model reflecting the mechanism of destruction clarified in the previous section as well as the corresponding method to identify parameters in the thermal model.

\subsection{Thermal equivalent circuit model}

Fundamentally, the proposed circuit model is based on the Cauer form. We separate the heat dissipation into the three major paths: the path through the supporting substrate, path through the package, and path through the lead frame. The detailed schematic is shown in Fig. 12. The thermal impedance $Z_{\text {thermal }}$ seen from the resistance body is expressed in Eq. (1), where the divided paths are formulated as Eqs. (2), (3), and (4), respectively. The variables in italics $R \mathrm{xx} C \mathrm{xx}$ stand for the values of the corresponding elements Rxx Cxx in Fig. 12. The copper (Cs3) on the rear side of the alumina substrates behaves as a heat spreader.

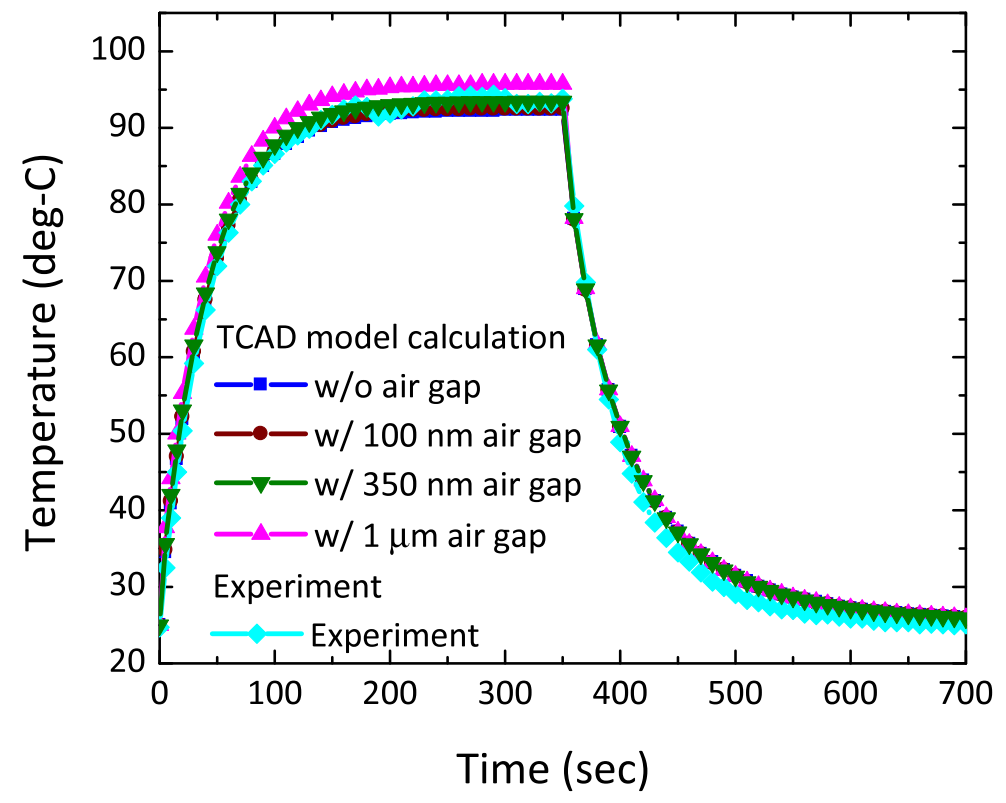

Fig. 9. Effective air gap width dependence of the long time thermal behavior of the resistors. 


$$
\begin{gathered}
Z_{\text {thermal }}=Z_{p} / / Z_{f} / / Z_{s} / / s C_{r 1} . \\
Z_{p}=R_{p 1}+\left(R_{p 2} / / \frac{1}{s C_{p 1}}\right)=R_{p 1}+\frac{1}{\frac{1}{R_{p 2}}+s C_{p 1}} . \\
Z_{f}=R_{f 1}+\left(R_{f 2} / / \frac{1}{s C_{f 1}}\right)=R_{f 1}+\frac{1}{\frac{1}{R_{f 2}}+s C_{f 1}} . \\
Z_{s}=R_{s 1}+\left(R_{s 2}+\left(R_{s 3}+\left(R_{s 4}+\left(R_{s 5}+\left(R_{s 6} / / \frac{1}{s C_{s 5}}\right) / /+\frac{1}{s C_{s 4}}\right) / /+\frac{1}{s C_{s 3}}\right) / /+\frac{1}{s C_{s 2}}\right) / /+\frac{1}{s C_{s 1}}\right) .
\end{gathered}
$$

\subsection{Thermal modeling methodology}

The proposed thermal modeling method essentially consists of the following three steps.

Step1. Reproduce measured thermal response by finite element method.

Step2. Identify thermal resistance corresponding to the convective boundaries.

Step3. Identify thermal RCs corresponding to the air gap colonies.

The first step is to reproduce the measured thermal response to the steady and pulsed power inputs, according to the TCAD model calculation with the finite element method [21]. We calibrate

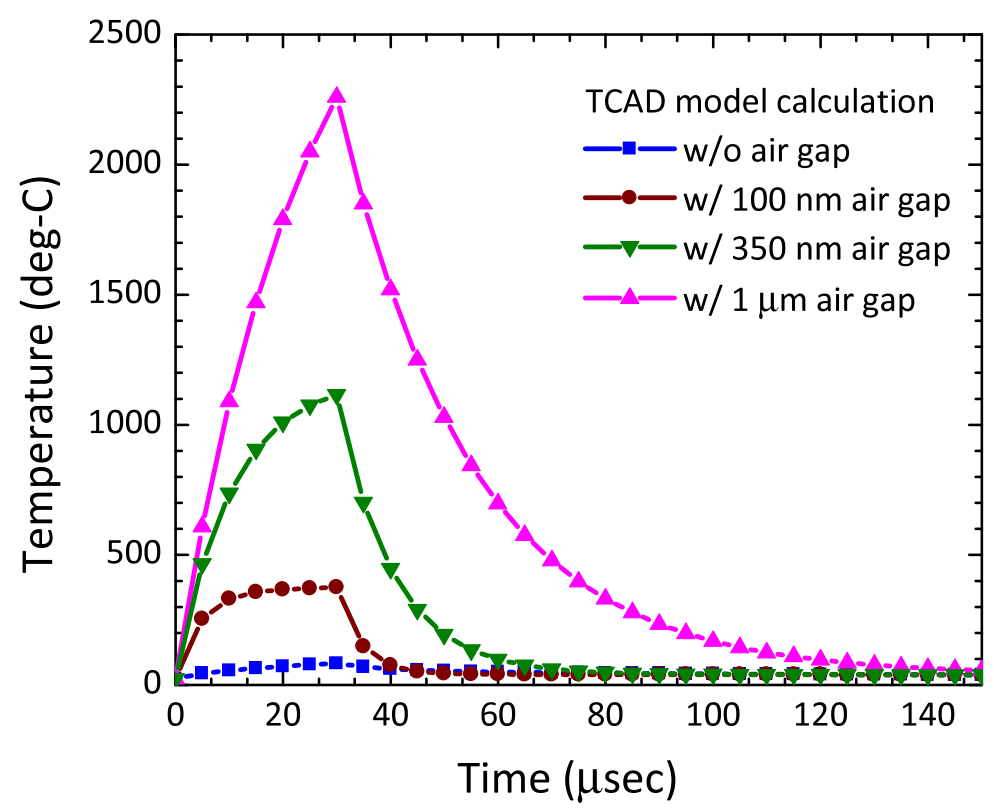

Fig. 10. Effective air gap width dependence of the short time thermal behavior of the resistors.

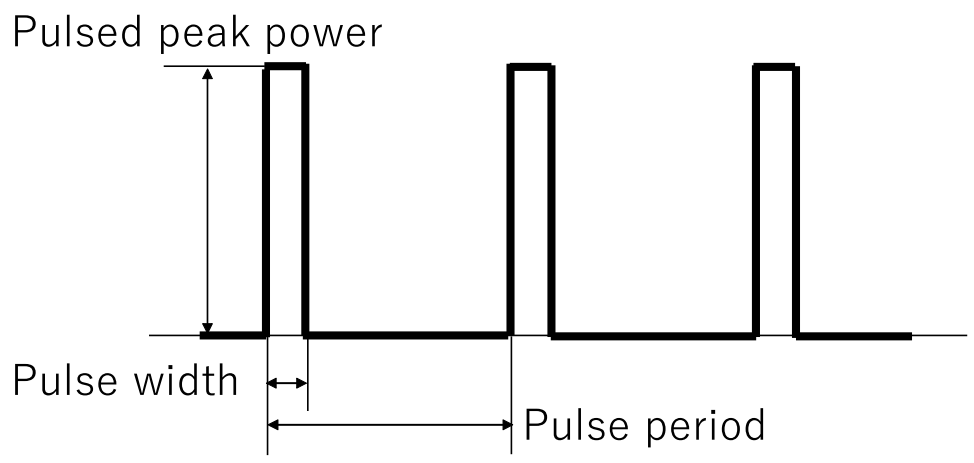

Fig. 11. Schematic of the incident pulsed power. 


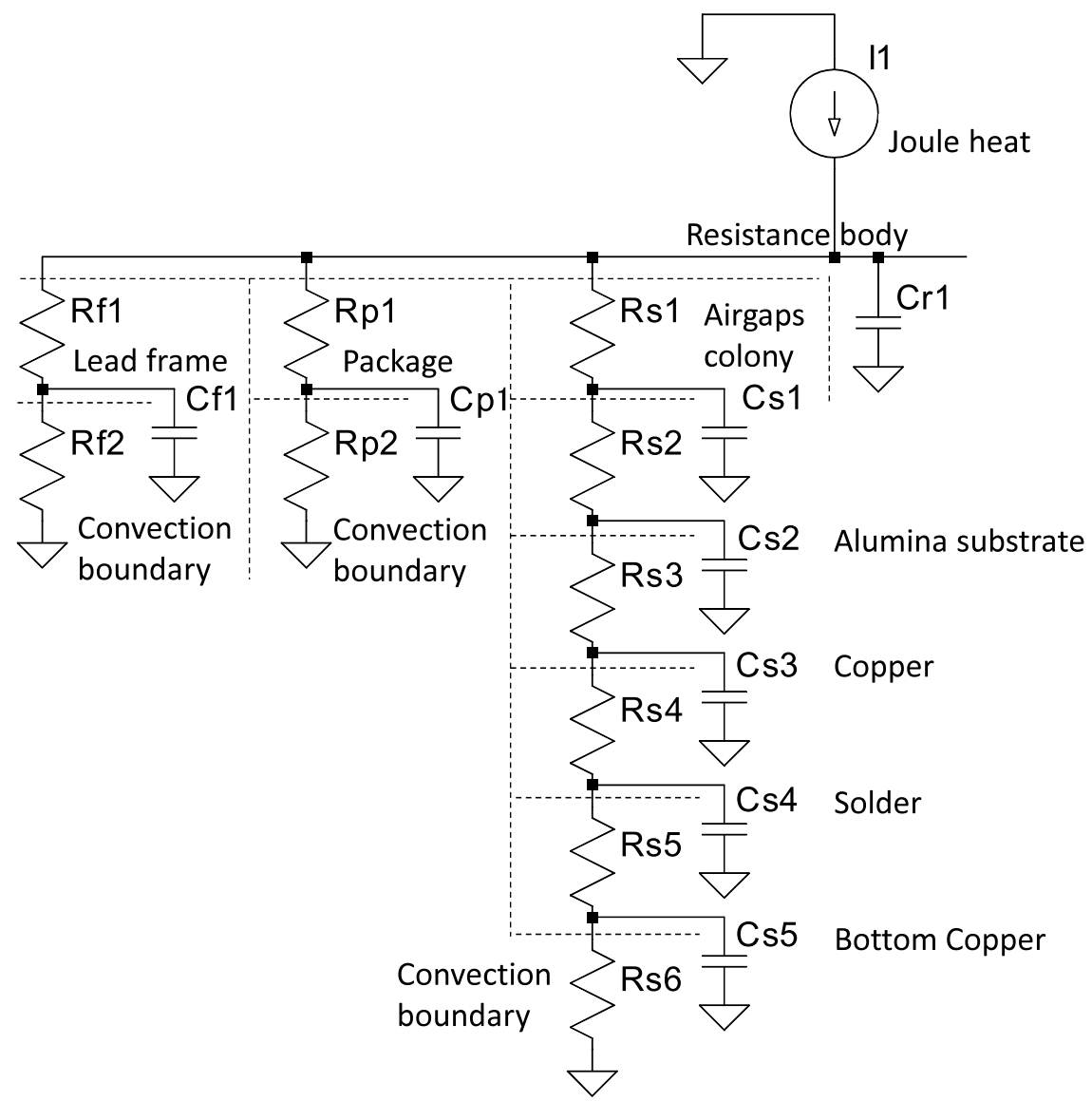

Fig. 12. Thermal equivalent circuit.

the convective boundary conditions based on the measured thermal response to the steady power input. The effective air gap is treated as a sheet (air sheet) in the TCAD calculation. By referring to the measured thermal response to the pulsed power input, we estimate the thickness of an air sheet so that the time constant caused by the air gap colony becomes equivalent to that of the air sheet.

The second and third steps are forming the thermal equivalent circuit depicted in Fig. 12.

The second step is to determine the values of thermal resistance $R_{f 2}, R_{p 2}$, and $R_{s 6}$, which correspond to the convective boundaries. In this step, we refer to the simulated thermal response to the steady power input. To identify each thermal resistance value, the rest of the boundaries are replaced with fixed boundaries.

The third step derives the values of thermal resistance $R_{s 1}$ and the thermal capacitance $C_{s 1}$, which correspond to the air gap colony. Here, we refer to the simulated thermal response to the pulsed power input. We determine $R_{s 1}$ to cause the highest temperature reached within a pulse width to reappear and then determine $C_{s 1}$ to fit the time constant of the simulated thermal response.

The rest of the thermal resistance and capacitance components are calculated from the dimensions and physical properties of the individual materials.

\section{Experimental results}

\subsection{Comparison of thermal behaviors between experiment and TCAD model calculation}

The input pulse width and pulse power dependence of the durability of the $\mathrm{NiCr}$ thin film resistors are considered. The TCAD model calculation results shown in Fig. 10 indicate that the existence of an effective air gap strongly affects the short thermal time constant and the reached temperature of the pulsed power injection. First, we determine the effective air gap width used for the TCAD model calculation. We compare the experimentally obtained destruction property with the TCAD model calculation results and evaluate the effective air gap width used for the following TCAD model 
(a)

\begin{tabular}{|c|c|c|c|c|}
\hline $\begin{array}{c}\text { Pulsed power } \\
\text { Pulse width- } \\
\text { Pulse period }\end{array}$ & $\begin{array}{c}30 \mu \mathrm{s}- \\
3 \mathrm{~ms}\end{array}$ & $\begin{array}{c}100 \mu \mathrm{s}- \\
10 \mathrm{~ms}\end{array}$ & $\begin{array}{c}300 \mu \mathrm{s}- \\
30 \mathrm{~ms}\end{array}$ & $\begin{array}{c}1 \mathrm{~ms}- \\
100 \mathrm{~ms}\end{array}$ \\
\hline $600 \mathrm{~W}$ & & & & \\
\hline $650 \mathrm{~W}$ & & & & \\
\hline $700 \mathrm{~W}$ & & & & \\
\hline $750 \mathrm{~W}$ & & & & \\
\hline $800 \mathrm{~W}$ & & & & \\
\hline $850 \mathrm{~W}$ & & & & \\
\hline $900 \mathrm{~W}$ & & & & \\
\hline $950 \mathrm{~W}$ & & & & \\
\hline $1000 \mathrm{~W}$ & & & & \\
\hline $1050 \mathrm{~W}$ & & & & \\
\hline $1100 \mathrm{~W}$ & & & & \\
\hline $1150 \mathrm{~W}$ & & & & \\
\hline $1200 \mathrm{~W}$ & & & & \\
\hline $1250 \mathrm{~W}$ & & & & \\
\hline $1300 \mathrm{~W}$ & & & & \\
\hline
\end{tabular}

(b)

\begin{tabular}{|c|c|c|c|c|}
\hline $\begin{array}{c}\text { Pulsed power } \\
\text { Pulse width- } \\
\text { Pulse period }\end{array}$ & $\begin{array}{c}30 \mu \mathrm{s}- \\
3 \mathrm{~ms}\end{array}$ & $\begin{array}{c}100 \mu \mathrm{s}- \\
10 \mathrm{~ms}\end{array}$ & $\begin{array}{c}300 \mu \mathrm{s}- \\
30 \mathrm{~ms}\end{array}$ & $\begin{array}{c}1 \mathrm{~ms}- \\
100 \mathrm{~ms}\end{array}$ \\
\hline $600 \mathrm{~W}$ & & & & \\
\hline $650 \mathrm{~W}$ & & & & \\
\hline $700 \mathrm{~W}$ & & & & \\
\hline $750 \mathrm{~W}$ & & & & \\
\hline $800 \mathrm{~W}$ & & & & \\
\hline $850 \mathrm{~W}$ & & & & \\
\hline $900 \mathrm{~W}$ & & & & \\
\hline $950 \mathrm{~W}$ & & & & \\
\hline $1000 \mathrm{~W}$ & & & & \\
\hline $1050 \mathrm{~W}$ & & & & \\
\hline $1100 \mathrm{~W}$ & & & & \\
\hline $1150 \mathrm{~W}$ & & & & \\
\hline $1200 \mathrm{~W}$ & & & & \\
\hline $1250 \mathrm{~W}$ & & & & \\
\hline $1300 \mathrm{~W}$ & & & & \\
\hline
\end{tabular}

Fig. 13. Resistor destruction power for different incident pulsed power and pulse width conditions. Shaded area represents the resistor destruction condition. (a) Experimental results and (b) TCAD model calculation results.

calculations. In this evaluation, we consider resistors without epoxy resin to simplify the analysis. In the analysis, we consider the sample destruction point for several different incident pulse width and incident pulse power conditions both in the experiment and in TCAD model calculation. In the TCAD model calculation, we judge that the sample is destructed when the temperature of the $\mathrm{NiCr}$ film exceeds the melting point of the $\mathrm{NiCr}$ around 1400 degrees in temperature.

Figure 13 shows the resistor destruction power for different incident pulsed power and pulse width conditions. In these figures, the shaded area represents that the samples are destructed. Figure 13(a) shows the experimental results and Fig. 13(b) shows the TCAD model calculation results. In Fig. 13(b), the effective air gap width introduced in the TCAD model calculation is $350 \mathrm{~nm}$. Here, to obtain the value of the effective air gap, we compare the results of the sample destruction power of the experiment with those of the TCAD model calculation.

Injected pulse width and pulsed peak power dependence of the resistor destruction power shown in Fig. 13(b) is strongly affected by the effective air gap width. Figure 10 suggests that, when the model without air gap is considered, temperature of the NiCr films does not change much for conditions of pulsed power injection. On the other hand, when the one micrometer width of air gap is assumed, the temperature is drastically increased within a quite short time. These behaviors indicate that appropriate effective air gap width exists to reproduce the experimental results in Fig. 13(a) in the TCAD model calculation. The experimentally obtained pulse destruction behavior (Fig. 13(a)) can be well reproduced by TCAD model calculation with $350 \mathrm{~nm}$ of effective air gap width condition (Fig. 13(b)). As a trend, when the pulse width is short, the durability of the resistors becomes high, and high pulsed power is needed to destruct the resistors. On the other hand, for the long pulse width conditions, a relatively small pulsed power can destruct the resistors. The TCAD model calculation can reproduce these experimentally obtained thermal behaviors well. From the above results, in the following of the paper the effective air gap introduced in the TCAD model calculation is set to $350 \mathrm{~nm}$.

Note that assuming the existence of an effective air gap in the TCAD model, the calculation results of the resistor destruction behavior in the incident pulsed power condition can well reproduce the experimental results. These behaviors are supporting evidence that the resistor destruction in pulsed power injection conditions originates from the thermal destruction due to the existence of the thermal isolation area in the $\mathrm{NiCr}$ thin films, and in the experiment, we considered that the samples locally overheat and melt. 
Table I. Identified thermal RC components.

\begin{tabular}{|c|c|c|c|c|c|c|}
\hline $\begin{array}{l}R_{f 1}(\mathrm{~K} / \mathrm{W}) \\
66.5\end{array}$ & $\begin{array}{l}C_{f 1}(\mathrm{~J} / \mathrm{K}) \\
0.6\end{array}$ & $\begin{array}{l}R_{p 1}(\mathrm{~K} / \mathrm{W}) \\
320\end{array}$ & $\begin{array}{l}C_{p 1}(\mathrm{~J} / \mathrm{K}) \\
0.42\end{array}$ & $\begin{array}{l}R_{s 1}(\mathrm{~K} / \mathrm{W}) \\
1.25\end{array}$ & $\begin{array}{l}C_{s 1}(\mathrm{~J} / \mathrm{K}) \\
3 e^{-3}\end{array}$ & $\begin{array}{l}C_{r 1}(\mathrm{~J} / \mathrm{K}) \\
10 e^{-6}\end{array}$ \\
\hline$R_{f 2}(\mathrm{~K} / \mathrm{W})$ & & $R_{p 2}(\mathrm{~K} / \mathrm{W})$ & & $R_{s 2}(\mathrm{~K} / \mathrm{W})$ & $C_{s 2}(\mathrm{~J} / \mathrm{K})$ & \\
\hline \multirow[t]{9}{*}{134} & & 60 & & 2 & 0.047 & \\
\hline & & & & $R_{s 3}(\mathrm{~K} / \mathrm{W})$ & $C_{s 3}(\mathrm{~J} / \mathrm{K})$ & \\
\hline & & & & $3.3 e^{-3}$ & $1.8 e^{-4}$ & \\
\hline & & & & $R_{s 4}(\mathrm{~K} / \mathrm{W})$ & $C_{s 4}(\mathrm{~J} / \mathrm{K})$ & \\
\hline & & & & 0.15 & 0.52 & \\
\hline & & & & $R_{s 5}(\mathrm{~K} / \mathrm{W})$ & $C_{s 5}(\mathrm{~J} / \mathrm{K})$ & \\
\hline & & & & $2.2 e^{-3}$ & $1.8 e^{-4}$ & \\
\hline & & & & $R_{s 6}(\mathrm{~K} / \mathrm{W})$ & & \\
\hline & & & & 100 & & \\
\hline
\end{tabular}
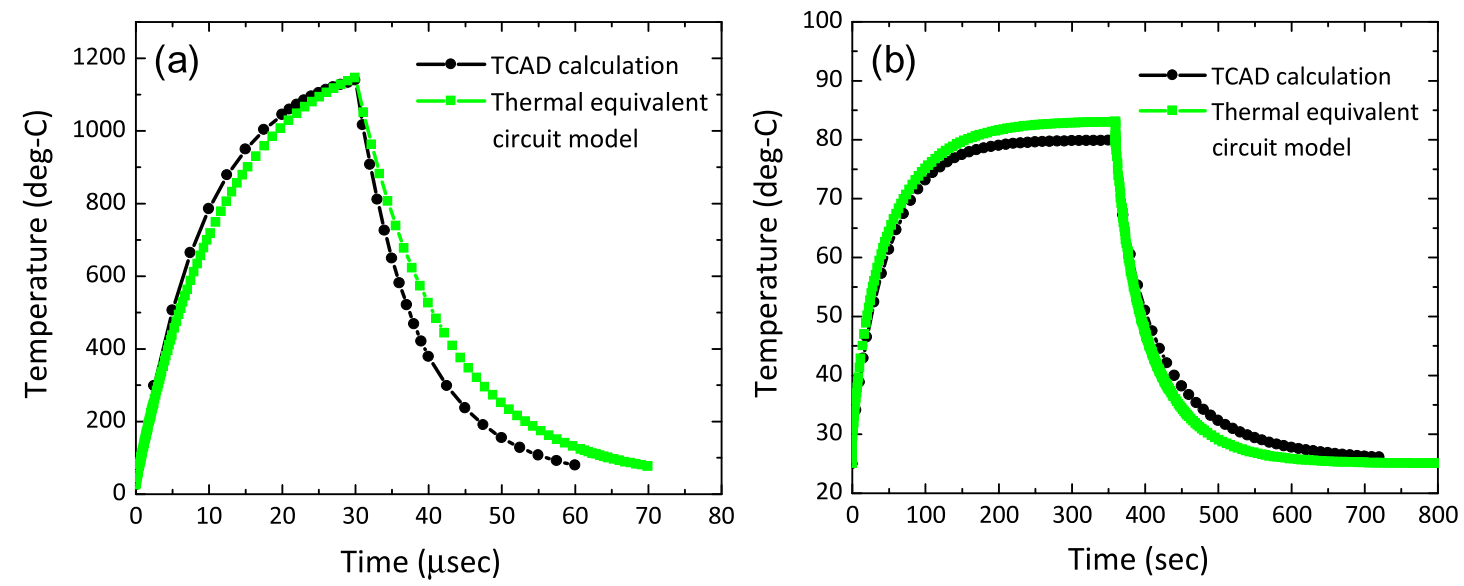

Fig. 14. Thermal behaviors of the resistors evaluated by the TCAD model calculation and the equivalent thermal circuit model. (a) Short time constant thermal behavior. $1000 \mathrm{~W}$ of pulsed power is applied for 30 microseconds. (b) Long time constant thermal behavior. $1 \mathrm{~W}$ of constant power is applied for 360 seconds.

\subsection{Comparison between TCAD model and thermal equivalent circuit model}

We compare the thermal behavior of the resistor obtained by the TCAD calculation and the thermal equivalent circuit model calculation to clarify the adequacy of the proposed thermal equivalent circuit model. The parameters shown in Table I are applied to the thermal circuit model and consider the thermal behaviors for different power and pulse width input conditions.

Initially, we fit the parameters of the thermal circuit model when the incident pulse width is 30 microseconds and pulse power is $1000 \mathrm{~W}$. Figure 14(a) indicates that both the calculation results agree well. Next, as shown in Fig. 14(b), a constant power of $1.0 \mathrm{~W}$ is applied for 360 seconds to consider the long time constant thermal behaviors, and this figure indicates that both the results also agree well. These figures show that the thermal behavior calculated by the TCAD model and the thermal circuit model agree well for both the short time constant (several tens of microseconds) and long time constant (several hundreds of seconds) situations.

Next, we evaluate the adequacy of the thermal circuit model by comparing the calculation results of the TCAD and thermal equivalent circuit model for a wide variety of pulse power and pulse width applied conditions. Figure 15(a)-(d) indicate that the results obtained by the thermal equivalent model can well reproduce the results of the TCAD model calculation. Also, Fig. 16 shows the resistor destruction power for different incident pulsed power and pulse width conditions. The shaded area represents that the samples are destructed. Figure 16(a) shows the experimental results and is the same as Fig. 13(a). Figure 16(b) shows the thermal equivalent circuit model results. The results obtained by thermal equivalent circuit model also reproduce the experimental results well. Based on the above results, the proposed thermal equivalent model in this study is adequately constructed, and it is useful to use this model for further numerical analysis because the thermal circuit model has the 

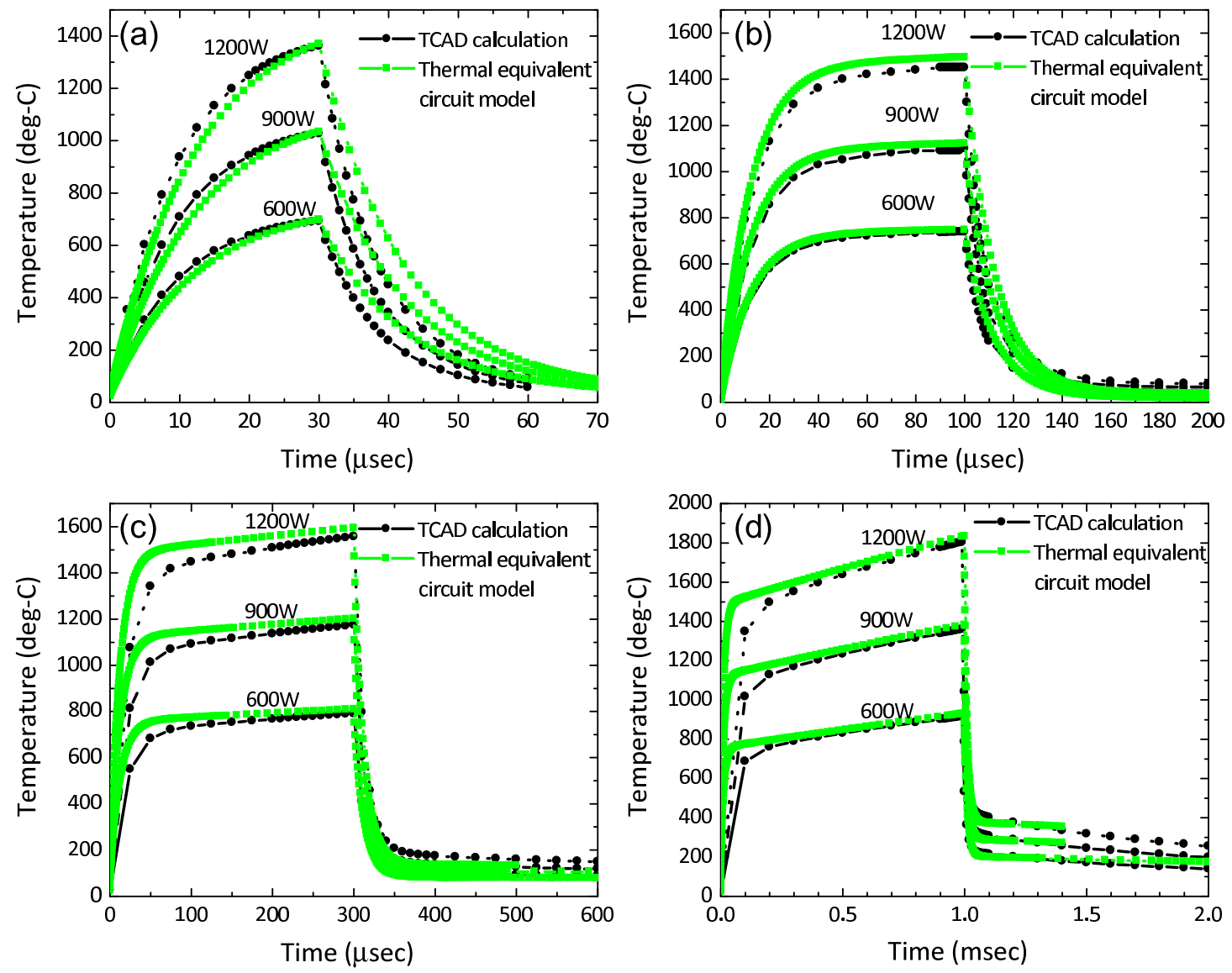

Fig. 15. Thermal behaviors of the resistors evaluated by the TCAD model calculation and the equivalent thermal circuit model for different pulsed power (600 W, $900 \mathrm{~W}$, and $12000 \mathrm{~W}$ ) applied conditions. (a) Pulsed power is applied for 30 microseconds. (b) Pulsed power is applied for 100 microseconds. (c) Pulsed power is applied for 300 microseconds. (d) Pulsed power is applied for $1 \mathrm{~ms}$.

(a)

\begin{tabular}{|c|c|c|c|c|}
\hline $\begin{array}{c}\text { Pulsed power } \\
\text { Pulse width- } \\
\text { Pulse period }\end{array}$ & $\begin{array}{c}30 \mu \mathrm{s}- \\
3 \mathrm{~ms}\end{array}$ & $\begin{array}{c}100 \mu \mathrm{s}- \\
10 \mathrm{~ms}\end{array}$ & $\begin{array}{c}300 \mu \mathrm{s}- \\
30 \mathrm{~ms}\end{array}$ & $\begin{array}{c}1 \mathrm{~ms}- \\
100 \mathrm{~ms}\end{array}$ \\
\hline $600 \mathrm{~W}$ & & & & \\
\hline $650 \mathrm{~W}$ & & & & \\
\hline $700 \mathrm{~W}$ & & & & \\
\hline $750 \mathrm{~W}$ & & & & \\
\hline $800 \mathrm{~W}$ & & & & \\
\hline $850 \mathrm{~W}$ & & & & \\
\hline $900 \mathrm{~W}$ & & & & \\
\hline $950 \mathrm{~W}$ & & & & \\
\hline $1000 \mathrm{~W}$ & & & & \\
\hline $1050 \mathrm{~W}$ & & & & \\
\hline $1100 \mathrm{~W}$ & & & & \\
\hline $1150 \mathrm{~W}$ & & & & \\
\hline $1200 \mathrm{~W}$ & & & & \\
\hline $1250 \mathrm{~W}$ & & & & \\
\hline $1300 \mathrm{~W}$ & & & & \\
\hline
\end{tabular}

(b)

\begin{tabular}{|c|c|c|c|c|}
\hline $\begin{array}{c}\text { Pulsed power } \\
\text { Pulse width- } \\
\text { Pulse period }\end{array}$ & $\begin{array}{c}30 \mu \mathrm{s}- \\
3 \mathrm{~ms}\end{array}$ & $\begin{array}{c}100 \mu \mathrm{s}- \\
10 \mathrm{~ms}\end{array}$ & $\begin{array}{c}300 \mu \mathrm{s}- \\
30 \mathrm{~ms}\end{array}$ & $\begin{array}{c}1 \mathrm{~ms}- \\
100 \mathrm{~ms}\end{array}$ \\
\hline $600 \mathrm{~W}$ & & & & \\
\hline $650 \mathrm{~W}$ & & & & \\
\hline $700 \mathrm{~W}$ & & & & \\
\hline $750 \mathrm{~W}$ & & & & \\
\hline $800 \mathrm{~W}$ & & & & \\
\hline $850 \mathrm{~W}$ & & & & \\
\hline $900 \mathrm{~W}$ & & & & \\
\hline $950 \mathrm{~W}$ & & & & \\
\hline $1000 \mathrm{~W}$ & & & & \\
\hline $1050 \mathrm{~W}$ & & & & \\
\hline $1100 \mathrm{~W}$ & & & & \\
\hline $1150 \mathrm{~W}$ & & & & \\
\hline $1200 \mathrm{~W}$ & & & & \\
\hline $1250 \mathrm{~W}$ & & & & \\
\hline $1300 \mathrm{~W}$ & & & & \\
\hline
\end{tabular}

Fig. 16. Resistor destruction power for different incident pulsed power and pulse width conditions. Shaded area represents the resistor destruction condition. (a) Experimental results (shown before). (b) Thermal equivalent model calculation results. 
merit that the thermal behaviors can be calculated with a small number of parameters.

\section{Conclusions}

In this research, the pulse durability of industrial thin film $\mathrm{NiCr}$ resistors were evaluated. We considered that the destruction behavior of the resistors for the pulsed power applied condition result from the thermal destruction of the $\mathrm{NiCr}$ thin films.

To analyze the sample destruction behavior of the pulse power applied situation, we proposed an equivalent thermal circuit model that mimics the thermal behaviors of the thin film $\mathrm{NiCr}$ resistors. Also, we performed TCAD model calculation to consider the thermal behaviors of the resistors. The experimental results and TCAD model calculation results of sample destruction conditions agree well when the effective air gap is introduced between the $\mathrm{NiCr}$ thin films and the alumina substrates. For the high pulsed power applied condition, the existence of the effective air gap thermal isolation layer leads to a drastic increase in the temperature of the thin $\mathrm{NiCr}$ film, and finally the NiCr films melt and destruct. When the effective air gap width is set to $350 \mathrm{~nm}$, the TCAD model calculation and the experimental results agree well. Also, the proposed equivalent thermal circuit model can reproduce the TCAD model calculation results for a wide range of applied pulsed power and applied pulse width conditions.

\section{Acknowledgments}

We would like to thank Dr. Shigeru Hidaka and Mr. Takuya Yamamichi of Nikkohm Co., Ltd. for their fruitful discussions. We also would like to thank Mr. Tsuneo Yoshioka and Ms. Chiyo Nakazawa of Nikkohm Co., Ltd. for useful advices.

\section{References}

[1] R.S. Chokhawala and S. Sobhani, "Switching voltage transient protection schemes for highcurrent IGBT modules," IEEE Transactions on Industry Applications, vol. 33, no. 6, pp. 16011610, November-December 1997.

[2] B.N. Torsaeter, S. Tiwari, R. Lund, and O. Midtgaard, "Experimental evaluation of switching characteristics, switching losses and snubber design for a full $\mathrm{SiC}$ half-bridge power module," Proc. 2016 IEEE 7th International Symposium on Power Electronics for Distributed Generation Systems (PEDG), pp. 1-8, 2016.

[3] H.S. Grover, F. Dawson, and E. Williams, "Impact of snubber circuit inductance on damping late currents in a pulsed power circuit," Proc. 2018 20th International Symposium on HighCurrent Electronics (ISHCE), pp. 130-134, 2018.

[4] K. Yatsugi, K. Nomura, and Y. Hattori, "Analytical technique for designing an RC snubber circuit for ringing suppression in a phase-leg configuration," IEEE Transactions on Power Electronics, vol. 33, no. 6, pp. 4736-4745, June 2018.

[5] J. vom Dorp, S.E. Berberich, T. Erlbacher, A.J. Bauer, H. Ryssel, and L. Frey, "Monolithic RCsnubber for power electronic applications," Proc. 2011 IEEE Ninth International Conference on Power Electronics and Drive Systems, pp. 11-14, 2011.

[6] R. Hata and S. Nishiyama, "The effect of built-in CR snubber capacitor into the power module," Proc. 2018 International Power Electronics Conference (IPEC), pp. 2149-2152, 2018.

[7] Y. Wang, Y. Li, X. Dai, S. Zhu, S. Jones, and G. Liu, "Thermal design of a dual sided cooled power semiconductor module for hybrid and electric vehicles," Proc. 2017 IEEE Applied Power Electronics Conference and Exposition (APEC), pp. 3068-3071, 2017.

[8] P.E. Bagnoli, C. Casarosa, M. Ciampi, and E. Dallago, "Thermal resistance analysis by induced transient (TRAIT) method for power electronic devices thermal characterization. I. Fundamentals and theory," IEEE Transactions on Power Electronics, vol. 13, no. 6, pp. 1208-1219, November 1998.

[9] P.E. Bagnoli, C. Casarosa, M. Ciampi, and E. Dallago, "Thermal resistance analysis by induced transient (TRAIT) method for power electronic devices thermal characterization. II. Practice 
and experiments," IEEE Transactions on Power Electronics, vol. 13, no. 6, pp. 1220-1228, November 1998.

[10] F. Kato, H. Nakagawa, H. Yamaguchi, and H. Sato, "Thermal resistance evaluation by hightemperature transient thermal analysis method for SiC power modules," Proc. 2016 International Conference on Electronics Packaging (ICEP), pp. 214-217, 2016.

[11] J. Yin, J.D. van Wyk, and W.G.H. Odendaal, "Comparison of transient thermal parameters for different die-connecting approaches," IEEE Transactions on Industry Applications, vol. 42, no. 6, pp. 1403-1411, November-December 2006.

[12] Z. Wang and W. Qiao, "A physics-based improved cauer-type thermal equivalent circuit for IGBT modules," IEEE Transactions on Power Electronics, vol. 31, no. 10, pp. 6781-6786, October 2016.

[13] T. Chen, S. Kuo, J. Hsu, and C. Pan, "Dynamic compact thermal modeling of package-onpackage by thermal resistor-capacitor ladder," Proc. 2016 15th IEEE Intersociety Conference on Thermal and Thermomechanical Phenomena in Electronic Systems (ITherm), pp. 223-229, 2016.

[14] J. Li, E. Deng, Z. Zhao, P. Zhang, and Y. Li, "Modelling the cauer thermal network for press pack IGBTs," Proc. 2017 Sixth Asia-Pacific Conference on Antennas and Propagation (APCAP), pp. 1-3, 2017.

[15] B. Chambers, T.Y. Tom Lee, and W. Blood, "Steady state and transient thermal analysis of chip scale packages," Proc. ITherm'98. Sixth Intersociety Conference on Thermal and Thermomechanical Phenomena in Electronic Systems (Cat. No.98CH36208), pp. 68-75, 1998.

[16] T. Evans, T. Hanada, Y. Nakano, and T. Nakamura, "Development of SiC power devices and modules for automotive motor drive use," Proc. 2013 IEEE International Meeting for Future of Electron Devices, pp. 116-117, 2013.

[17] K.R. Bagnall, Y.S. Muzychka, and E.N. Wang, "Application of the Kirchhoff Transform to Thermal Spreading Problems With Convection Boundary Conditions," IEEE Transactions on Components, Packaging and Manufacturing Technology, vol. 4, no. 3, pp. 408-420, March 2014.

[18] S.I. Shkuratov, M. Kristiansen, J.C. Dickens, L.L. Hatfield, and E. Horrocks, "High-current and high-voltage pulsed testing of resistors," IEEE Transactions on Plasma Science, vol. 28, no. 5, pp. 1607-1614, October 2000.

[19] A. Klossowicz, P. Winiarski, and A. Dziedzic, "Pulse durability of thin-film resistors embedded in printed circuit boards," Proc. 2011 International Students and Young Scientists Workshop "Photonics and Microsystems", pp. 68-72, 2011.

[20] D.P. Muffoletto et al., "Thermodynamic modeling with experimental validation of the pulsed and periodic operation of a high power resistor," Proc. 2011 IEEE Pulsed Power Conference, pp. 239-244, 2011.

[21] P.M. Souare et al., "Thermal correlation between measurements and FEM simulations in 3D ICs," Proc. 2013 IEEE International 3D Integration Conference (3DIC), pp. 1-6, 2013.

[22] FEMTET Version 2018.1., Murata Software Co., Ltd., https://www.muratasoftware.com/en/. 